\title{
THE SOLAR DYNAMO
}

\author{
D. SCHMITT \\ Universitäts-Sternwarte, Geismarlandstr. 11 \\ D-3400 Göttingen, Federal Republic of Germany
}

\begin{abstract}
The generation of the solar magnetic field is generally ascribed to dynamo processes in the convection zone. The dynamo effects, differential rotation $(\omega$-effect $)$ and helical turbulence $(\alpha-$ effect) are explained, and the basic properties of the mean-field dynamo equations are discussed in close comparison with the observed solar cycle.

Especially the question of the seat of the dynamo is addressed. Problems of a dynamo in the convection zone proper could be magnetic buoyancy, the nearly strict observance of the polarity rules and the migration pattern of the magnetic fields which are difficult to understand in the light of recent studies of the field structure in the convection zone and by observations of the solar acoustic oscillations. To overcome some of these problems it has been suggested that the solar dynamo operates in the thin overshoot region at the base of the convection zone instead. Some aspects of such an interface dynamo are discussed. As an alternative to the turbulent $\alpha$-effect a dynamic $\alpha$-effect based on magnetostrophic waves driven by a magnetic buoyancy instability of a magnetic flux layer is introduced. Model calculations for both pictures, a convection zone and an interface dynamo, are presented which use the internal rotation of the sun as deduced from helioseismology. Solutions with solar cycle behaviour are only obtained if the magnetic flux is bounded in the lower convection zone and the $\alpha$-effect is concentrated near the equator.

Another aspect briefly addressed is the nonlinear saturation of the magnetic field. The necessity of the dynamic nature of the dynamo processes is emphasized, and different processes, e.g. magnetic buoyancy and $\alpha$-quenching, are mentioned.
\end{abstract}

Key words: Mean-Field Electrodynamics - Dynamo Theory - Sun

\section{Introduction}

With a kinematic turbulent $\alpha \omega$-dynamo the global properties of the solar magnetic field can be reasonably well described. Such a dynamo consists of two basic elements:

a) differential rotation or $\omega$-effect, which produces a toroidal field by continuously winding up a poloidal field, and

b) the $\alpha$-effect which is an induction effect of rotating turbulent matter and regenerates the poloidal field component.

The latter effect is crucial for a dynamo. As a consequence of cyclonic motions it was first qualitatively described by Parker (1955). Within the framework of mean-field electrodynamics which was established by Steenbeck, Krause and Rädler (1966) the effect of small-scale motions on large-scale magnetic fields has been systematically investigated.

By placing the dynamo in the convection zone and making reasonable assumptions about these two effects, many features of the solar cycle can be well represented (e.g. Steenbeck \& Krause 1969 and many others, see review by Rädler 1990) like Maunder's butterfly diagram, Hale's polarity rules and the phase relations between the field components.

This general agreement of the calculated fields with the observed patterns provided confidence that the basic ideas are correct. The hope was that minor disagreements could be resolved with a better knowledge of the solar differential rotation 
and a realistic turbulence model for the mean electromotive force, i.e. the $\alpha$-effect and turbulent diffusivity.

However, recent observations of the surface magnetic field and the $\mathrm{p}$-mode oscillations as well as theoretical considerations about the field structure within the convection zone have seriously put this picture into question.

\section{Magnetic Field Structure and Dynamo Theory in the Convection Zone}

Investigations in magnetoconvection (reviewed in Galloway \& Weiss 1981 and Proctor \& Weiss 1982) suggest that the vast majority of the solar magnetic flux in the convection zone is concentrated in small-scale intermittent features as observed on the solar surface (Stenflo 1973).

These features are difficult to store in the convection zone for times comparable to the solar cycle. A couple of processes, namely magnetic buoyancy (Parker 1975, Schüssler 1977, Moreno-Insertis 1983), the passive transport of small structures by convection (e.g. Schüssler 1987), convective and hydrodynamical instabilities and fragmentation processes (Schüssler 1979a, Tsinganos 1980, Spruit \& van Ballegooijen 1982, van Ballegooijen 1983, van Ballegooijen \& Choudhuri 1988, Schüsssler 1990, Ferriz-Mas \& Schüssler 1992) transports magnetic flux from the bottom to the top of the convection zone in times of the order of one month, much too short for the dynamo to generate the field.

A second problem of locating the dynamo within the convection zone is the nearly strict observance of the polarity rules for bipolar active regions. Golub et al. (1981) argued the small-scale magnetic fields associated with X-ray bright points and ephemeral active regions, which do not show polarity preferences and solar cycle dependence, originate in the convection zone and are disorganized due to turbulent shredding and tangling. The magnetic flux, however, that emerges as bipolar active regions aligned nearly parallel to the equator provides evidence of an underlying field that originates in a distinctly less turbulent region than the convection zone itself. Such a region would be more likely to have fields that obey Hale's polarity rules. Moreover, the field must possess sufficient strength in order to avoid distorsion.

These problems grow even more severe as helioseismology shows with increasing reliability that differential rotation does not at all dominate over convective motions in the convection zone proper. The oscillation data imply that the main convection zone rotates like the solar surface with no significant radial gradient, and that the deep interior rotates almost rigidly at a rate intermediate between the equatorial and polar rates on the surface (Brown \& Morrow 1987, Libbrecht 1988, Dziembowski et al. 1989, Brown et al. 1989, Schou et al. 1992). Thus a radial gradient occurs in a transitional region between the bottom of the convection zone and the top of the interior. Schüssler (1984) had already proposed this radial gradient. His argument was based on the gradient of the intensity of turbulence which causes a net transport of momentum towards the base of the convection zone.

An $\alpha \omega$-dynamo in the convection zone with only a latitudinal gradient of angular velocity results in a stationary solution for $\alpha<0$ or in an oscillatory solution 
for $\alpha>0$ in the northern hemisphere. In the latter case the dynamo wave proceeds radially outward (Yoshimura 1975) from the bottom to the top of the convection zone with diffusion acting also in latitudinal direction. The resulting butterfly diagram is not solar-like. Most of the flux is concentrated at too high latitudes and there is no clear latitudinal migration (Köhler 1973, Prautzsch 1993). The period is much too short.

A possible alternative is a dynamo with an anisotropic $\alpha$-tensor (e.g. Rüdiger 1978, Rädler 1980). Weisshaar (1982) presented an $\alpha^{2}$-dynamo model with a solarlike behaviour (butterfly diagram, period) with anisotropies $\alpha_{r r}<0, \alpha_{\theta \theta}=\alpha_{\varphi \varphi}>$ $0, \alpha_{r \theta}=\alpha_{\theta r}>0$ and $\left|\alpha_{r r}\right|>\left|\alpha_{\varphi \varphi}\right|$. At that time the author considered his $\alpha-$ tensor as rather unrealistic. It is however interesting to notice that recent turbulence models yield just such an anisotropic $\alpha$-tensor (Wälder et al. 1980, Brandenburg et al. 1990, Rüdiger \& Kichatinov 1992, Ferrière 1992). Brandenburg \& Tuominen's (1988) $\alpha^{2} \omega$-dynamo model with anisotropies should not be forgotten in this context. Elstner \& Rüdiger (this volume) however report, that non-axisymmetric $(m=1)$ modes are preferred with such an anisotropic $\alpha$-tensor, which again is in conflict with the observed properties of the solar magnetic field.

A completely different picture of a convection zone dynamo has been proposed by Schüssler (1980 and this volume), which is, due to the field structure in the convection zone, based on statistics of flux tubes rather than on an averaged field.

\section{Aspects of a Dynamo at the Base of the Convection Zone}

\subsection{Overshoot REgION}

Part of the problems mentioned above can be released if the source region of the magnetic flux, which emerges in active regions, and the site of the dynamo is the slightly subadiabatic region of overshooting convection between the convection zone proper and the radiative interior.

In the overshoot layer of a depth of some $10^{4} \mathrm{~km}$ (van Ballegooijen 1982, Schmitt et al. 1984, Pitadella \& Stix 1986, Skaley \& Stix 1991) large amplitude fields of $10^{4}$ $\mathrm{G}$ to $10^{5} \mathrm{G}$ can be stored for times of the order of the cycle (Moreno-Insertis et al. 1992). Sunspots are due to rised flux tubes that are still firmly anchored in this layer (Moreno-Insertis 1986).

It has been proposed for these and a couple of other reasons that the bulk of the solar magnetic field is stored in the convective overshoot region at the base of the convection zone (e.g. Spiegel \& Weiss 1980, Schüssler 1983, Hughes 1991). This layer is also the favourable site for the solar dynamo. There the radial gradient of angular velocity yields dynamo waves migrating in latitudinal direction, there occurs a dynamic $\alpha$-effect which is acting on a strong toroidal field, and there magnetic diffusivity is likely to be reduced so that the cycle period increases. These points will be taken up in more detail in the next sections.

\subsection{Differential Rotation}

The already mentioned results from solar acoustic oscillations say that the radial gradient of angular velocity at the base of the convection zone changes its sign at a 
latitude of about $30^{\circ}$. The gradient is positive near the equator and negative near the pole. At the pole the gradient seems to be steeper approximately by a factor of two. There is still a latitudinal gradient which is somewhat smaller than at the surface.

If one confines the dynamo to the overshoot region by reducing the turbulent diffusivity and confining the $\alpha$-effect there with $\alpha \sim \cos \theta$, a positive $\alpha$ in the northern hemisphere yields oscillatory magnetic fields which occur at high latitudes migrating towards the equator (Prautzsch 1993). Near the equator one obtains a hint of a branch migrating towards the pole. A negative $\alpha$ would change the migration in the desired direction but yields a stationary solution. This is because of the effect of $\partial \Omega / \partial \theta$. Without this gradient one would obtain an oscillatory solution with an equatorial branch migrating towards the equator which is associated with the butterfly diagram of sunspots, and a polar branch of filaments migrating poleward (Schmitt 1987, Gilman et al. 1989, Prautzsch 1990). Since $|\partial \Omega / \partial r|$ is larger near the pole, the polar branch dominates in contrast to the case of the sun.

\section{3. $\alpha$-Effect of Magnetostrophic Waves}

The second and most important ingredient of a dynamo is the $\alpha$-effect. Overshooting convection provides a negative $\alpha$ in the northern hemisphere (Yoshimura 1972, Glatzmaier $1985 \mathrm{a}, \mathrm{b})$. This usually results in the stationary $\alpha \omega$-dynamo solutions mentioned above. The effectivity of this only kinematicly explored effect in uncertain.

The energy density of the overshoot layer magnetic field is comparable to or larger than the kinetic energy density of the convective motions. Further the filling factor of magnetic flux is likely to be large. This means that a kinematic approach would be inadequate and a dynamic dynamo is required.

Fortunately the intense toroidal magnetic field layer of the overshoot region provides an alternative dynamic $\alpha$-effect (Schmitt 1984, 1985, 1987). The top parts of the layer, where the magnetic field decreases rapidly enough with height, are unstable due to magnetic buoyancy. This is a Rayleigh-Taylor like instability where the potential energy of extra mass supported against gravity, is released by downward transport of mass and upward transport of magnetic flux. Because of the solar rotation the instability takes the form of growing magnetostrophic waves (Acheson \& Hide 1973, Acheson \& Gibbons 1978, Acheson 1978, 1979, Schmitt 1985). These are helical and are therefore capable of inducing an electromotive force parallel to the toroidal field (Schmitt 1984, 1985). This force drives an electric current which regenerates the poloidal field (Schmitt 1987). This dynamic $\alpha$-effect is applicable to strong fields because the velocity is not prescribed but follows from the present forces and the interaction of the magnetic field with the velocity field is taken into account. It is, by the way, not based on convection. A similar effect due to flux tube instabilities is currently investigated (Schüssler et al. 1993).

Superimposing the most unstable magnetostrophic waves an $\alpha$-effect is derived which depends non-monotonicly on latitude. $\alpha$ is antisymmetric with respect to the equator where it vanishes. In the northern hemisphere it is negative from the equator to a latitude of approximately $30^{\circ}$ where it changes sign and is slightly 
positive thereafter. Therefore $\alpha$ is concentrated mainly at lower latitudes (Schmitt 1985).

With such an $\alpha$-effect the above stationary solution of an overshoot layer dynamo turns oscillatory (Prautzsch 1993) (Fig. 1). The magnetic field is now generated only in a small region around the equator at the bottom of the convection zone. A butterfly diagram plotted there would show a migration of dynamo waves towards the equator with too many belts overlapping each other. Near the top of the convection zone nice butterfly diagrams of the there much weaker fields are derived which are similar to Maunder's sunspot diagrams. Also the period of oscillation is of the order of the solar cycle. At the present this seems to be the only solution with solar cycle behaviour.

A polar branch is not seen. This much weaker branch has observational evidence in the behaviour of filaments (Stix 1974) and polar faculae (Makarov \& Makarova 1986 ) and is seen in magnetograms (Howard \& LaBonte 1981, Stenflo 1988) and in coronal activity (Leroy \& Noens 1983). It might be a surface effect (Topka et al. 1982, DeVore \& Sheeley 1987, Sheeley et al. 1987, Wang et al. 1991, Durney et al. 1993 ) since it is not present in the observed torsional oscillations which occur first near the poles, migrate in the course of 22 years to the equator (Howard \& LaBonte 1980 ) and are interpreted as a consequence of the magnetic field on solar rotation (Schüssler 1981, Yoshimura 1981, Rüdiger et al. 1986, Rüdiger \& Kichatinov 1990).

\subsection{Phase Relations}

A problem of overshoot layer dynamos is the phase relation between the toroidal and radial field components (Stix 1976, 1987). Observationally they are completely out of phase. With $\partial \Omega / \partial r>0$ near the equator an equatorward migration of the dynamo wave is obtained for $\alpha<0$. Then however the questioned field components are in phase. The observed phase relation can only be obtained if the signs of both differential rotation and $\alpha$ are reversed. There are a couple of possibilities to resolve this difficulty. The measurements are tricky and the observations might be not reliable. There might be different phase relations at the bottom and the top of the convection zone. Anisotropies and fluctuations of the $\alpha$-tensor as well as nonlinearities might have an effect on the phase relation. Not only for this reason the effect of reasonable anisotropic $\alpha$-tensors on dynamo models in the overshoot region needs to be investigated.

\subsection{Magnitudes of InduCtion EFFECTS}

For a dynamo operating in a thin layer Choudhuri (1990) estimated the magnitudes of the induction effects needed in order to reproduce the period and latitudinal wavelength of the solar cycle. He found that the dynamo should be of $\alpha^{2} \omega$ type (Gilman et al. 1989), the radial shear should be of the order of $G=\partial v_{\varphi} / \partial r \approx$ $10^{-5} \mathrm{~s}^{-1} \ldots 10^{-6} \mathrm{~s}^{-1}$, values consistent with the results from acoustic oscillations, the $\alpha$-effect of magnitude $\alpha \approx 10 \mathrm{cms}^{-1}$ (Köhler 1973, Schmitt 1984), and the magnetic diffusivity of $\eta \approx 10^{10} \mathrm{~cm}^{2} \mathrm{~s}^{-1}$. When interpreting the latter two values as turbulent eddy values, a rms velocity of $30 \mathrm{~ms}^{-1}$ and a quite small value of $300 \mathrm{~km}$ for the 


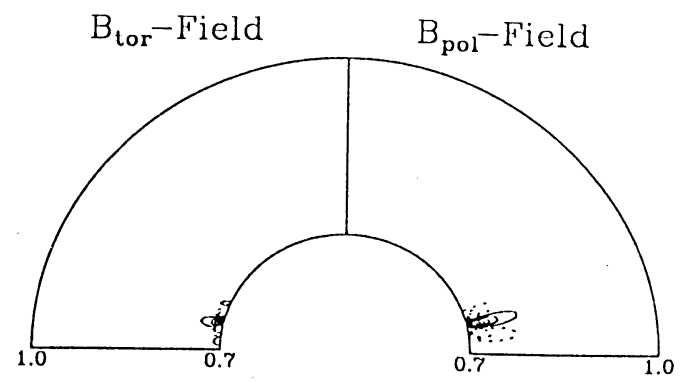

$\mathrm{B}_{\text {tor }}-$ Field
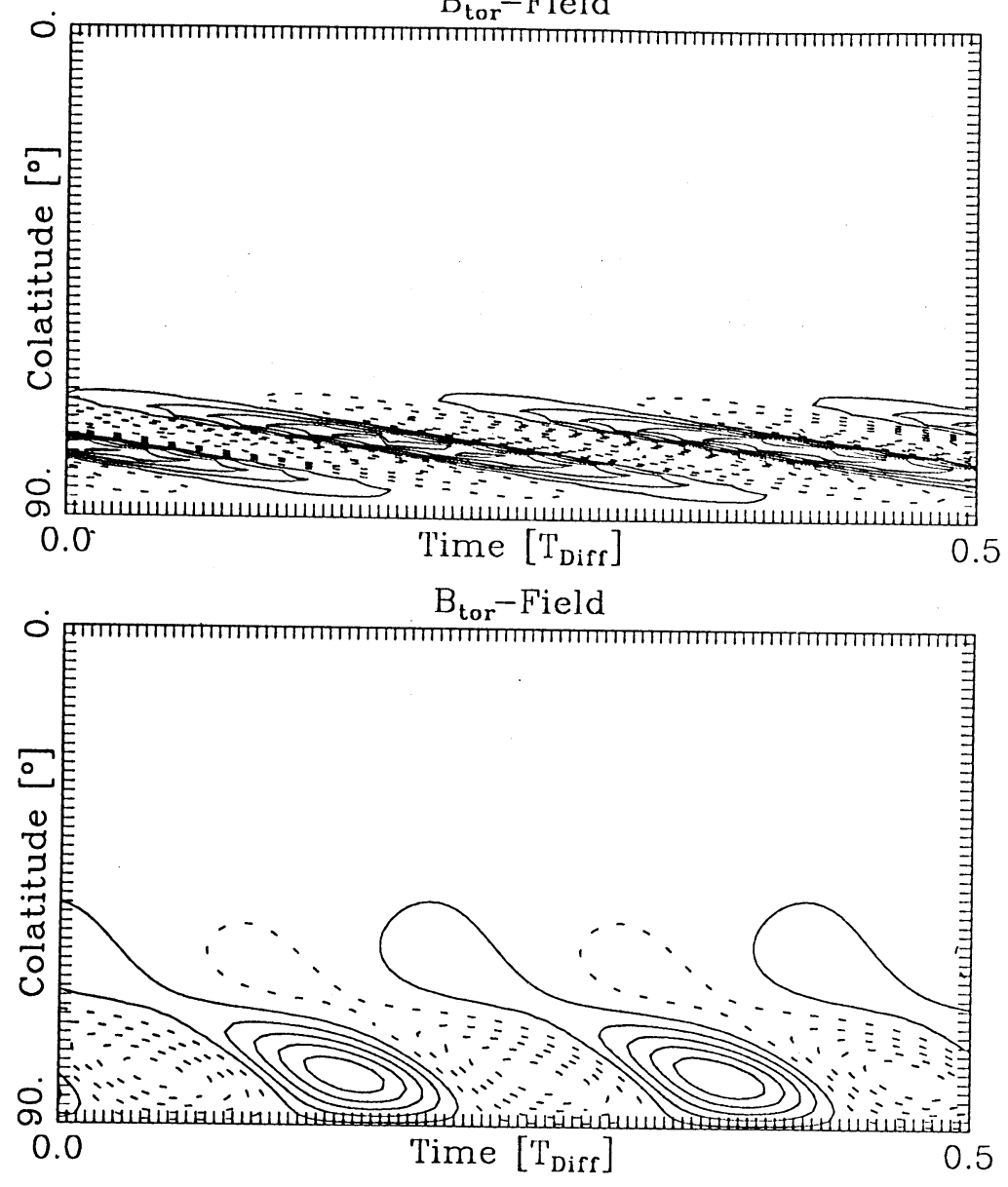

Fig. 1. Overshoot layer $\alpha^{2} \omega$-dynamo with differential rotation as deduced from helioseismology, reduced magnetic diffusivity at the lower boundary and $\alpha$ concentrated in the overshoot region and within $\pm 30^{\circ}$ around the equator: Snapshot of the toroidal and poloidal field components (top panel) and butterfly diagrams of the toroidal field component near the bottom (middle panel) and near the top (bottom panel) of the convection zone. 
turbulent length scale would result.

\section{Nonlinear Models}

A complete solar dynamo must include the backreaction of the magnetic field upon the material motion by the Lorentz force which stabilizes the amplitude of the generated magnetic field. This means the solution of the MHD equations. In view of the expected high field strength in the overshoot region such a dynamic approach is really necessary. Since it however means serious complications and efforts the nonlinear feedback of the field upon the motions is often treated in parametrized forms.

Flux escape by magnetic buoyancy is probably a dominating ingredient of the nonlinear limitation of the solar dynamo. Various parametrized descriptions (Leighton 1969, Robinson \& Durney 1982, DeLuca \& Gilman 1986, Schmitt \& Schüssler 1989, Jennings \& Weiss 1991) have been adopted, the most advanced is by Moss et al. (1990a,b) who added a magnetic field dependent velocity, directed radially outward, to the induction equation, thought as the rise velocity of buoyant magnetic flux tubes. With such a description weak buoyancy limits the field strength of the above favored oscillatory solution. A beat phenomenon and asymmetries between successive half-cycles (reported in Moss et al. 1990a) fade away with sufficient numerical resolution. With strong buoyancy however the oscillatory solution disappears and the stationary mode is obtained (Prautzsch, private communication). Due to the gradient of the turbulence intensity turbulent diamagnetism (Zel'dovich 1956) and topological pumping (Drobyshevski \& Yuferev 1974), which represent a drift of the mean magnetic field ( $\gamma$-effect) (Rädler 1968) towards the bottom of the convection zone (Schüssler 1984, Kichatinov 1991, Brandenburg et al. 1992, Schmitt 1993), counteract magnetic buoyancy in the overshoot region and lower convection zone in favour of the oscillatory mode.

A strong magnetic field interfers with the small-scale motions which give rise to the $\alpha$-effect. A growing field amplitude leads to a decrease of the $\alpha$-effect $(\alpha-$ quenching). For a turbulence model with a distributed turbulence intensity but neglected density stratification Rüdiger \& Kichatinov (1992) provide quenching functions for all components of the $\alpha$-tensor and for an arbitrary strength of the magnetic field. A whole variety of dynamo models has been presented based on an ad-hoc fashion of this nonlinearity (e.g. Schmitt \& Schüssler 1989, Brandenburg et al. 1989, Rädler et al. 1990, Jennings \& Weiss 1991) with complex behaviour like exchange of stability and symmetry breaking at bifurcations, mixed parity solutions, and dependencies on initial conditions.

The large-scale mean field generated by the dynamo process drives a mean flow described by the momentum equation. According to Lenz rule, the induction effect of this flow, meridional circulation and differential rotation, counteracts its source, the mean field, and thus represents a field limiting mechanism and leads to finite amplitude magnetic fields in $\alpha$-effect dynamos (Malkus \& Proctor 1975, Proctor 1977, Hellmich 1978, Schüssler 1979b, Fearn \& Proctor 1984, 1987, Brandenburg et al. 1989). Quenching of differential rotation alone, as a special case of this mechanism, has been approximated by Yoshimura (1978), Hinata (1982) and Belvedere et 
al. (1990). DeLuca and Gilman $(1986,1988)$ present a model especially for the overshoot region. Probably the most advanced mean-field dynamo is by Brandenburg et al. $(1991,1992)$ with differential rotation generated by the $\Lambda$-effect (Rüdiger 1989). This is somewhat in between pure kinematic models and full 3D simulations without refering to the mean-field concept (Gilman \& Miller 1981, 1986, Gilman 1983, Glatzmaier 1984). As in the latter the results are however not satisfactory in the light of the observed properties of the solar cycle (differential rotation and butterfly diagram).

A fascinating feature of nonlinear dynamo models is the possibility of chaotic solutions which occur in truncated systems similar to the Lorenz equations. I refer to the investigations of Weiss et al. (1984), Belvedere et al. (1990), Meinel \& Brandenburg (1990), Schmalz \& Stix (1991) and Kurths et al. (this volume). Irregular behaviour like global minima in the solar cycle was found. There is however the suspicion that simpler behaviour would prevail if the truncation was less severe.

The possibility of the stochastic excitation of magnetic fields by a fluctuating $\alpha$-effect has been put forward by Hoyng $(1987,1988,1990)$. This may contribute to irregularities in the solar cycle (Choudhuri 1992, Moss et al. 1992) and leads to the excitation of a whole spectrum of dynamo modes (Teuben et al. 1993), which can be compared with the decomposition of magnetogram fields into spherical harmonics (Stenflo \& Vogel 1986, Stenflo \& Weisenhorn 1987, Stenflo \& Güdel 1988, Stenflo 1988). A similar analysis based on sunspots was recently presented by Gokhale et al. (1990, 1992) and Gokhale \& Javaraiah (1990, 1992). These investigations yield many quantitative data which can be used as diagnostic tools to tune parameters in an inversion dynamo problem (Schmitt 1993).

\section{Conclusion}

A satisfying solar dynamo model is still missing. The original hope that detailed observational and theoretical information would yield better results of the dynamo did not prove true, on the contrary, they raised difficulties instead.

With the differential rotation deduced from solar acoustic oscillations one cornerstone of dynamo theory is established. A consistent $\alpha$-tensor and diffusivity from a detailed turbulence model is desirable and information how they are affected by the magnetic field (quenching) (Rüdiger \& Kichatinov 1992). The overshoot layer dynamo has to be explored in more detail, especially the most likely field limiting process of magnetic buoyancy has to be added to the models (see also Moss et al. 1990b).

I have only briefly mentioned the effect of nonlinearities like magnetic buoyancy and $\alpha$-quenching. Also other important aspects related to the solar dynamo (turbulent transport, stability, symmetry, dynamics, $\alpha$-fluctuations, flux tube dynamo, ...), partly not touched at all, deserve more interest. Many of these points are however taken up in other papers of this volume (Kichatinov, Hoyng, Elstner, Schüssler, Brandenburg, Weiss, Kurths, ...). Textbooks on dynamo theory are those of Moffatt (1978), Krause \& Rädler (1980) and Parker (1979), while more recent developments on the solar dynamo are reviewed by Stix (1987), Weiss (1989), Rädler (1990), Hoyng (1990), Brandenburg \& Tuominen (1991), DeLuca \& Gilman (1991) 
and $\operatorname{Stix}(1991)$.

\section{Acknowledgements}

I would like to thank T. Prautzsch for allowing me to present numerical results before publication and W. Deinzer, T. Prautzsch and M. Schüssler for valuable discussions.

\section{References}

Acheson, D.J.: 1978, Phil. Trans. Roy. Soc. A289, 459

Acheson, D.J.: 1979, Solar Phys. 62, 23

Acheson, D.J. \& Hide, R.: 1973, Rep. Prog. Phys. 36, 159

Acheson, D.J. \& Gibbons, M.P.: 1978, J. Fluid Mech. 85, 743

Belvedere, G., Pitadella, R.M. \& Proctor, M.R.E.: 1990, Geophys. Astrophys. Fluid Dyn. 51, 263

Brandenburg, A. \& Tuominen, I.: 1990, 'The Solar Dynamo', in The Sun and Cool Stars: activity, magnetism, dynamos, Tuominen, I., Moss, D. \& Rüdiger, G. (eds.), IAU-Coll. 130, Lecture Notes in Physics 380, 223

Brandenburg, A., Krause, F., Meinel, R., Moss, D. \& Tuominen, I.: 1989, Astron. Astrophys. 213, 411

Brandenburg, A., Nordlund, A., Pulkinnen, P., Stein, R.F. \& Tuominen, I.: 1990, Astron. Astrophys. 232, 277

Brandenburg, A., Moss, D., Rüdiger, G. \& Tuominen, I.: 1991, Geophys. Astrophys. Fluid Dyn. 61, 179

Brandenburg, A., Moss, D. \& Tuominen, I.: 1992, 'Stratification and thermodynamics in meanfield dynamos', preprint

Brandenburg, A., Moss, D. \& Tuominen I.: 1992, 'Turbulent pumping in the solar dynamo', in NSO/Sacramento Peak 12th Summer Workshop, Harvey, K.L. (ed.), Astron. Soc. Pacific Conf. Proc.

Brown, T.M., Morrow, C.A.: 1987, Astrophys. J. 314, L21

Brown, T.M., Christensen-Dalsgaard, J., Dziembowski, W.A., Goode, P., Gough, D.O. \& Morrow, C.A.: 1989, Astrophys. J. 343, 526

Choudhuri, A.R.: 1990, Astrophys. J. 355, 733

Choudhuri, A.R.: 1992, Astron. Astrophys. 253, 277

DeLuca, E.E. \& Gilman, P.A.: 1986, Geophys. Astrophys. Fluid Dyn. 37, 85

DeLuca, E.E. \& Gilman, P.A.: 1988, Geophys. Astrophys. Fluid Dyn. 43, 119

DeLuca, E.E. \& Gilman, P.A.: 1991, 'The Solar Dynamo', in The Solar Atmosphere and Interior, Cox, A.N. (ed.), Univ. of Arizona Press, Tucson

DeVore, C.R. \& Sheeley, N.R., Jr.: 1987, Solar Phys. 108, 47

Drobyshevski, E.M. \& Yuferev, V.S.: 1974, J. Fluid Mech. 65, 33

Durney, B.R., DeYoung, D.S. \& Roxburgh, I.W.: 1993, 'On the generation of the large-scale and turbulent magnetic fields in solar-type stars', Solar Phys., in press

Dziembowksi, W.A., Goode, P.R. \& Libbrecht, K.G.: 1989, Astrophys. J. 337, L53

Elstner, D. \& Rüdiger, G.: 1993, 'Stellar dynamos with anisotropic $\alpha$-tensor', this volume

Fearn, D.R. \& Proctor, M.R.E.: 1984, Phys. Earth Planet. Inter, 36, 78

Fearn, D.R. \& Proctor, M.R.E.: 1987, J. Fluid Mech. 178, 521

Ferrière, K.: 1992, 'The Full Alpha-Tensor Due to Supernova Explosions and Superbubbles in the Galactic Disk', preprint

Ferriz-Mas, A., Schüssler, M.: 1992, 'Instabilites of magnetic flux tubes in a stellar convection zone. I: Toroidal flux tubes in differentially roatating stars', preprint

Galloway, D.J. \& Weiss, N.O.: 1981, Astrophys. J. 243, 945

Gilman, P.A.: 1983, Astrophys. J. Suppl. 53, 243

Gilman, P.A., Miller, J.: 1981, Astrophys. J. Suppl. 46, 211

Gilman, P.A., Miller, J.: 1986, Astrophys. J. Suppl. 61, 585

Gilman, P.A., Morrow, C.A. \& DeLuca, E.E.: 1989, Astrophys. J. 338, 528

Glatzmaier, G.A.: 1984, J. Comp. Phys. 55, 461 
Glatzmaier, G.A.: 1985a, Astrophys. J. 291, 300

Glatzmaier, G.A.: 1985b, Geophys. Astrophys. Fluid Dyn. 31, 137

Gokhale, M.H. \& Javaraiah, J.: 1990, Mon. Not. Royal Astron. Soc. 243, 241

Gokhale, M.H. \& Javaraiah, J.: 1992, Solar Phys. 138, 399

Gokhale, M.H., Javaraiah, J. \& Hiremath, K.M.: 1990, in Solar Photosphere: Structure, Convection and Magnetic Fields, Stenflo, J.O. (ed.), IAU-Symp. 138, 375

Gokhale, M.H., Javaraiah, J., Narayanan Kutty, K. \& Varghese, B.A.: 1992, Solar Phys. 138,35

Golub, L., Rosner, R., Vaiana, G.S. \& Weiss, N.O.: 1981, Astrophys. J. 243, 309

Hellmich, R.: 1978, Geophys. Astrophys. Fluid Dyn. 10, 89

Hinata, S.: 1982, Astrophys. J. 256, L23

Howard, R.F. \& LaBonte, B.J.: 1980, Astrophys. J. 239, L33

Howard, R.F. \& LaBonte, B.J.: 1991, Solar Phys. 74, 131

Hoyng, P.: 1987, Astron. Astrophys. 171, 348 and 357

Hoyng, P.: 1988, Astrophys. J. 332, 857

Hoyng, P.: 1990, 'Excitation of Dynamo Waves', in Solar Photosphere: Structure, Convection and Magnetic Fields, Stenflo, J.O. (ed.), IAU-Symp. 138, 359

Hughes, D.W.: 1991, 'Magnetic Buoyancy', in Advances in Solar System Magnetohydrodynamics, Priest, E.R. \& Wood, A.W. (eds.), Cambridge University Press, p. 77

Jennings, R.L. \& Weiss, N.O.: 1991, Mon. Not. R. Astr. Soc. 252, 249

Kichatinov, L.L.: 1991, Astron. Astrophys. 243, 483

Köhler, H.: 1973, Astron. Astrophys. 25, 467

Krause, F. \& Rädler, K.-H.: 1980, Mean-Field Magnetohydrodynmics and Dynamo Theory, Pergamon Press, Oxford

Kurths, J., Feudel, U. \& Brandenburg, A.: 1993, 'Inhomogeneous chaos in nonlinear dynamo models', this volume

Leighton, R.B.: 1969, Astrophys. J. 156, 1

Leroy, J.-L. \& Noens, J.-C.: 1983, Astron. Astrophys. 120, L1

Libbrecht, K.G.: 1988, 'Solar p-mode frequency splittings', in Seismology of the Sun and Sun-Like Stars, Rolfe, E.J. (ed.), ESA SP-286, p. 131

Makarov, V.I. \& Makarova, V.V.: 1986, J. Astrophys. Astron. 7, 113

Malkus, W.V.R. \& Proctor, M.R.E.: 1975, J. Fluid Mech. 67, 417

Meinel, R. \& Brandenburg, A.: 1990, Astron. Astrophys. 238, 369

Moffatt, H.K.: 1978, Magnetic Field Generation in Electrically Conducting Fluids, Cambridge University Press

Moreno-Insertis, F.: 1983, Astron. Astrophys. 122, 241

Moreno-Insertis, F.: 1986, Astron. Astrophys. 166, 291

Moreno-Insertis, F., Ferriz-Mas, A. \& Schüssler, M.: 1992, 'Storage of magnetic flux tubes in a convective overshoot region', Astron. Astrophys., in press

Moss, D., Brandenburg, A., Tavakol, R. \& Tuominen, I.: 1992, 'Stochastic effects in mean field dynamos', preprint

Moss, D., Tuominen, I. \& Brandenburg, A.: 1990a, Astron. Astrophys. 228, 284

Moss, D., Tuominen, I. \& Brandenburg, A.: 1990b, Astron. Astrophys. 240, 142

Parker, E.N.: 1955, Astrophys. J. 122, 293

Parker, E.N.: 1975, Astrophys. J. 198, 205

Parker, E.N.: 1979, Cosmical Magnetic Fields, Clarendon Press, Oxford

Pitadella, R.M. \& Stix, M.: 1986, Astron. Astrophys. 157, 338

Prautzsch, T.: 1990, 'Der Einfluß von breitenabhängigen Induktionseffekten auf einen eindimensionalen sphärischen $\alpha \omega$-Dynamo', Diplomarbeit, Universität Göttingen

Prautzsch, T.: 1993, 'The Dynamo Mechanism in the Deep Convection Zone of the Sun', in NATO ASI Theory of Solar and Planetary Dynamos, Proceedings of the Isaac Newton Institute, Cambridge

Prautzsch, T., Schmitt, D. \& Schüssler, M: 1993, 'Non-linear dynamos. II. Two-dimensional model of an overshoot layer dynamo', in preparation

Proctor, M.R.E.: 1977, J. Fluid Mech. 80, 769

Proctor, M.R.E. \& Weiss, N.O.: 1982, Rep. Prog. Phys. 45, 1317

Rädler, K.-H.: 1968, Zs. Naturforsch. 23a, 1851

Rädler, K.-H.: 1980, Astron. Nachr. 301, 101

Rädler, K.-H.: 1990, 'The Solar Dynamo', in Inside the Sun, Berthomieu G. \& Cribier M. (eds.), 
Kluwer, Dordrecht, IAU-Coll. 121, 385

Rädler, K.-H., Wiedemann, E., Brandenburg, A., Meinel, R. \& Tuominen, I.: 1990, Astron. Astrophys. 239, 413

Robinson, R.D. \& Durney, B.R.: 1982, Astron. Astronphys. 108, 322

Rüdiger, G.: 1978, Astron. Nachr. 299, 217

Rüdiger, G.: 1989, Differential Rotation and Stellar Convection, Akademie-Verlag Berlin

Rüdiger, G. \& Kichatinov, L.L.: 1990, Astron. Astrophys. 236, 503

Rüdiger, G. \& Kichatinov, L.L.: 1992, 'Alpha-effect and alpha-quenching', preprint

Rüdiger, G., Tuominen, I., Krause, F. \& Virtanen, H.: 1986, Astron. Astrophys. 166, 306

Schmalz, S. \& Stix, M.: 1991, Astron. Astrophys. 245, 654

Schmitt, D.: 1984, 'Dynamo Action of Magnetostrophic Waves', in The Hydromagnetics of the Sun, Guyenne, T.D., Hunt, J.J. (eds.), ESA SP-220, 223

Schmitt, D.: 1985, 'Dynamowirkung magnetostrophischer Wellen', Thesis, Universität Göttingen

Schmitt, D.: 1987, Astron. Astrophys. 174, 281

Schmitt, D. \& Schüssler, M.: 1989, Astron. Astrophys. 223, 343

Schmitt, D.: 1993, 'Inversion approach to the solar dynamo', in preparation

Schmitt, D.: 1993, 'The influence of turbulent diamagnetism and topological pumping on an $\alpha \omega-$ dynamo in the deep convection zone of the sun', in preparation

Schmitt, J.H.M.M., Rosner, R. \& Bohn, H.U.: 1984, Astrophys. J. 282, 316

Schou, J., Christen-Dalsgaard, J. \& Thompson, M.J.: 1992, Astrophys. J. 385, L59

Schüssler, M.: 1977, Astron. Astrophys. 56, 439

Schüssler, M.: 1979a, Astron. Astrophys. 71, 79

Schüssler, M.: 1979b, Astron. Astrophys. 72, 348

Schüssler, M.: 1980, Nature 288, 150

Schüssler, M.: 1981, Astron. Astrophys. 94, L17

Schüssler, M.: 1983, 'Stellar Dynamo Theory', in Solar and Stellar Magnetic Fields: Origins and Coronal Effects, Stenflo, J.O. (ed.), IAU-Symp. 102, 213

Schüssler, M.: 1984, 'On the Structure of Magnetic Fields in the Solar Convection Zone', in The Hydromagnetics of the Sun Guyenne, T.D., Hunt, J.J. (eds.), ESA SP-220, 67

Schüssler, M.: 1987, 'Magnetic Fields and the Rotation of the Solar Convection Zone', in The Internal Solar Angular Velocity, Durney, B.R. \& Sofia, S. (eds.), Reidel, Dordrecht, p. 303

Schüssler, M.: 1990, 'Comments on the structure and dynamics of magnetic fields in stellar convection zones', Habilitationschrift, Universität Göttingen

Schüssler, M., Ferriz-Mas, A. \& Schmitt, D.: 1993, 'An $\alpha$-effect due to flux tube instabilities in the solar convection zone', in preparation

Sheeley, N.R., Nash, A.G. \& Wang, Y.-M.: 1987, Astrophys. J. 319, 481

Skaley, D. \& Stix, M.: 1991, Astron. Astrophys. 241, 227

Spiegel, E.A. \& Weiss, N.O.: 1980, Nature 287, 616

Spruit, H.C. \& van Ballegooijen, A.A.: 1982, Astron. Astrophys. 106, 58

Steenbeck, M. \& Krause, F.: 1969, Astron. Nachr. 291, 49

Steenbeck, M., Krause, F. \& Rädler, K.-H.: 1966, Z. Naturforsch. 21a, 369

Stenflo, J.O.: 1973, Solar Phys. 32, 41

Stenflo, J.O.: 1988, Astrophys. Space Sci. 144, 321

Stenflo, J.O. \& Güdel, M.: 1988, Astron. Astrophys. 191, 137

Stenflo, J.O. \& Vogel, M.: 1986, Nature 319, 285

Stenflo, J.O. \& Weisenhorn, A.L.: 1987, Solar Phys. 108, 205

Stix, M.: 1974, Astron. Astrophys. 37, 121

Stix, M.: 1976, Astron. Astrophys. 47, 243

Stix, M.: 1987, 'On the Origin of Stellar Magnetism', in Solar and Stellar Physics, Schröter, E.-H. \& Schüssler, M. (eds.), Lecture Notes in Physics, 292, 15

Stix, M.: 1991, Geophys. Astrophys. Fluid Dyn. 62, 211

Teuben, L., Hoyng, P. \& Schmitt, D.: 1993, 'Periodicities in the Large-Scale Solar Magnetic Field', in preparation

Topka, K., Moore, R., LaBonte, B.J. \& Howard, R.: 1982, Solar Phys. 79, 231

Tsinganos, K.C.: 1980, Astrophys. J. 239, 746

van Ballegooijen, A.A.: 1982, Astron. Astrophys. 113, 99

van Ballegooijen, A.A.: 1983, Astron. Astrophys. 118, 275

van Ballegooijen, A.A. \& Choudhuri, A.R.: 1988, Astrophys. J. 333, 965 
Wädler, M., Deinzer, W. \& Stix, M.: 1980, J. Fluid Mech. 96, 207

Wang, Y.-M., Sheeley, N.R. \& Nash, A.G.: 1991, Astrophys. J. 383, 431

Weiss, N.O.: 1989, 'Dynamo Processes in Stars', in Accretion Discs and Magnetic Fields in Astrophysics, Belvedere, G. (ed.), Kluwer, Dordrecht, p. 11

Weiss, N.O.: 1990, Phil. Trans. R. Soc. Lond. A330, 617

Weiss, N.O., Cattaneo, F. \& Jones, C.A.: 1984, Geophys. Astrophys. Fluid Dyn. 30, 305

Weisshaar, E.: 1982, Geophys. Astrophys. Fluid Dyn. 21, 285

Yoshimura, H.: 1972, Astrophys. J. 178, 863

Yoshimura, H.: 1975, Astrophys. J. 201, 740

Yoshimura, H.: 1978, Astrophys. J. 220, 692

Yoshimura, H.: 1981, Astrophys. J. 247, 1102

Zel'dovich, Ya.B.: 1956, Sov. Phys. JETP 31, 154 